\title{
Exact Analytical Solution of the Peristaltic Nanofluids Flow in an Asymmetric Channel with Flexible Walls and Slip Condition: Application to the Cancer Treatment
}

\author{
Abdelhalim Ebaid ${ }^{1}$ and Emad H. Aly ${ }^{2,3}$ \\ ${ }^{1}$ Department of Mathematics, Faculty of Science, University of Tabuk, Tabuk 71491, Saudi Arabia \\ ${ }^{2}$ Department of Mathematics, Faculty of Science, King Abdulaziz University, Jeddah 21589, Saudi Arabia \\ ${ }^{3}$ Department of Mathematics, Faculty of Education, Ain Shams University, Roxy, Cairo 11757, Egypt \\ Correspondence should be addressed to Emad H. Aly; emad-aly@hotmail.com
}

Received 31 May 2013; Accepted 3 August 2013

Academic Editor: Gabriel Turinici

Copyright (C) 2013 A. Ebaid and E. H. Aly. This is an open access article distributed under the Creative Commons Attribution License, which permits unrestricted use, distribution, and reproduction in any medium, provided the original work is properly cited.

In the cancer treatment, magnetic nanoparticles are injected into the blood vessel nearest to the cancer's tissues. The dynamic of these nanoparticles occurs under the action of the peristaltic waves generated on the flexible walls of the blood vessel. Studying such nanofluid flow under this action is therefore useful in treating tissues of the cancer. In this paper, the mathematical model describing the slip peristaltic flow of nanofluid was analytically investigated. Exact expressions were deduced for the temperature distribution and nano-particle concentration. In addition, the effects of the slip, thermophoresis, and Brownian motion parameters on the temperature and nano-particle concentration profiles were discussed and further compared with other approximate results in the literatures. In particular, these results have been obtained at the same values of the physical examined parameters that was considered in Akbar et al., "Peristaltic flow of a nanofluid with slip effects," 2012. The results reveal that remarkable differences are detected between the exact current results and those approximately obtained in the literatures for behaviour of the temperature profile and nano-particles concentration. Accordingly, the current analysis and results are considered as optimal and therefore may be taken as a base for any future comparisons.

\section{Introduction}

In the recent times, peristalsis has attracted much attention due to its important engineering and medical applications, like chyme movement in the intestine, movement of eggs in the fallopian tube, transport of the spermatozoa in cervical canal, transport of bile in the bile duct, transport of cilia, circulation of blood in small blood vessels and in the intrauterine fluid flow within the uterine cavity. Since the first investigation of Latham [1], several theoretical and experimental studies have been conducted to understand peristaltic action [1-11]. In particular, to describe peristaltic flow in a symmetric channel or axisymmetric tubes containing Newtonian or nonNewtonian fluids, many models have been investigated by Zien and Ostrach [2], Lee and Fung [3], Srivastava et al. [4], El Shehawey and Mekheimer [6],
Ramachandra and Usha [7], and Mekheimer and Abd elmaboud [10].

Further, present application is in the embryo transfer within the uterine cavity, where physiologists observed that the intra-uterine fluid flow due to myometrial contractions is peristaltic-type motion. In addition, De Vries et al. [12] found that the myometrial contractions may occur in both symmetric and asymmetric directions. Therefore, a great effort was devoted to study the peristaltic flow of Newtonian and nonNewtonian fluids in an asymmetric channel [13-23], and recently of Jeffrey and Johnson-Segalman fluids; see, for example, [24-28].

Although a huge number of studies for the peristaltic flow of classical fluids are available, only few papers are available for the peristaltic flow of nanofluids [29-34]. In this regard, Akbar et al. [33] may be the first authors to study the 
peristaltic nanofluids, in which the peristaltic flow and heat transfer of a nanofluid in an asymmetric channel have been analyzed. They have used the homotopy perturbation method to obtain the analytical approximate solutions for the temperature distribution and the nanoparticles concentration.

The nanofluids are a new class of fluids designed by dispersing nanometer-sized materials (nanoparticles, nanofibers, nanotubes, nanowires, nanorods, nanosheet, or droplets) in base fluids. Choi [35] reported that an innovative technique to improve heat transfer is by using nanoscale particles in the base fluid. Further, Choi et al. [36] showed that the addition of a small amount (less than $1 \%$ by volume) of nanoparticles to conventional heat transfer liquids increased the thermal conductivity of the fluid up to approximately two times.

In the tumors treatment, one of the effective methods is to inject the blood vessel nearest to the tumor with magnetic nanoparticles along with placing a magnet close to the tumor. These particles act like heat sources, in the presence of the applied magnetic field of alternating nature. Mekheimer and Abd elmaboud [10] found that the cancer's tissues are destroyed if the temperature reaches $42-45^{\circ} \mathrm{C}$. On the other hand, in this application the drug may be placed on the magnetic nanoparticles and is injected near the tumor. Then, the drug is absorbed by the tumor through a high gradient magnetic field, which is concentrated near the tumor center [37]. Regarding, Habibi et al. [38] mentioned that the drug absorption due to high concentration of the magnetic particles increases and magnetic force prevents uniform drug distribution in circulatory system. This approach reduces the side effect and allows using high dose of anticancer drug. It should be noted that Majumder et al. [39] indicated that nanofluidic flow usually exhibits partial slip against the solid surface, which can be characterized by the so-called slip length, around 3.4-68 $\mathrm{mm}$ for different liquids.

The aim of this paper is to declare the exact effects of the slip, thermophoresis, and Brownian motion parameters on the temperature and nanoparticle concentration profiles of nanofluid flow in an asymmetric channel. It is well known that the exact solution of any physical model is optimal when available and would lead to the correct physical interpretations of the involved phenomena. Therefore, an approach is presented to achieve this goal for the resulting system of linear and nonlinear partial differential equations derived by Akbar et al. [33]. Then, these exact solutions are invested in obtaining the correct behaviour of the physical quantities.

\section{The Mathematical Investigated Model}

In the current work, we consider the peristaltic transport of an incompressible Newtonian nanofluid in an asymmetric channel with flexible walls, generating by propagation of waves on the channel walls traveling with different amplitudes and phases but with the same constant speed $c$. In the Cartesian coordinates system $(x, y)$, the upper wall $h_{1}$ and lower wall $h_{2}$ are given by, see Figure 1,

$$
h_{i}=A d_{i}+a_{i} \cos \left[\frac{2 \pi}{\lambda}(x-c t)+B \varphi\right]
$$

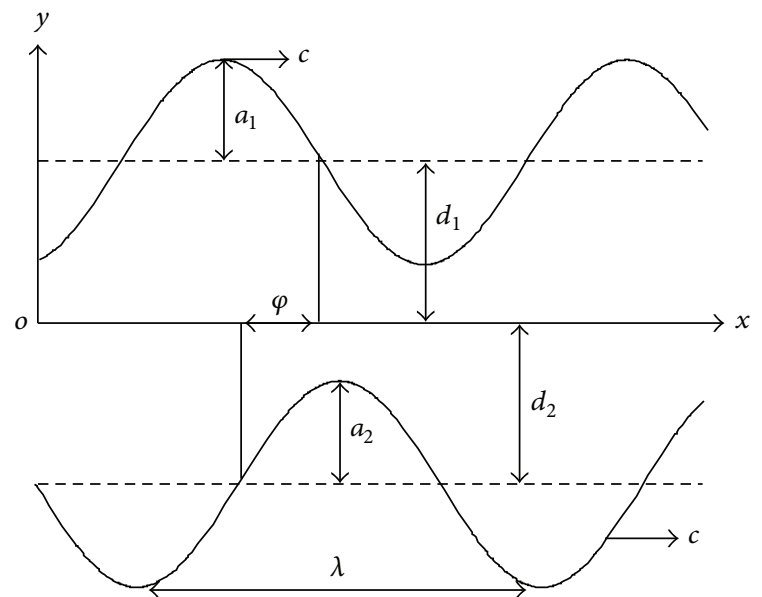

FIGURE 1: Schematic diagram of a two-dimensional asymmetric channel.

where $A=1,-1$ and $B=0,1$ when $i=1,2$, respectively. Further, $a_{1}$ and $a_{2}$ are the amplitude of the waves, $\lambda$ is the wave length, $d_{1}+d_{2}$ is the width of the channel, the phase difference $\varphi$ varies in the range $0 \leq \varphi \leq \pi$, where $\varphi=0$, and $\pi$ corresponds to symmetric channel with waves out of the phase and in the phase, respectively. It should be noted that the following condition has to be achieved [40]:

$$
a_{1}^{2}+a_{2}^{2}+2 a_{1} a_{2} \cos \varphi \leq\left(d_{1}+d_{2}\right)^{2}
$$

with the following nondimensional phenomena [33]:

$$
a=\frac{a_{1}}{d_{1}}, \quad b=\frac{a_{2}}{d_{1}}, \quad d=\frac{d_{2}}{d_{1}} .
$$

On considering heat transfer along with nanoparticles phenomena under the assumptions of long wavelength and low Reynolds number approximation, Akbar et al. [33] found that the flow is governed by the following system of partial differential equations:

$$
\begin{gathered}
\psi_{y y y y}+G_{r} \theta_{y}+\beta_{r} \sigma_{y}=0, \\
\theta_{y y}+N_{b} \theta_{y} \sigma_{y}+N_{t}\left(\theta_{y}\right)^{2}=0, \\
\sigma_{y y}+\frac{N_{t}}{N_{b}} \theta_{y y}=0, \\
\frac{d p}{d x}=\left(\psi_{y y}+G_{r} \theta+\beta_{r} \sigma\right)_{y}
\end{gathered}
$$

where $\psi, \theta, \sigma$, and $p$ are the stream function, temperature distribution, nanoparticles concentration, and pressure gradient, respectively. In addition, $N_{b}, N_{t}, G_{r}$, and $B_{r}$ are the Brownian motion parameter, thermophoresis parameter, local temperature Grashof number, and nanoparticles Grashof number, respectively. The system (4)-(6) has to be 
solved subject to the following boundary conditions on $\psi, \theta$, and $\sigma$ :

$$
\begin{gathered}
\psi=\frac{F}{2}, \quad \psi_{y}=-\beta \psi_{y y}-1 \quad \text { at } h_{1}=1+a \cos (x) \\
\psi=-\frac{F}{2}, \quad \psi_{y}=\beta \psi_{y y}-1 \quad \text { at } h_{2}=-d-b \cos (x+\varphi) \\
\theta+\gamma \theta_{y}=0, \quad \text { at } y=h_{1} \\
\theta-\gamma \theta_{y}=1, \quad \text { at } y=h_{2} \\
\sigma+\gamma_{1} \sigma_{y}=0, \quad \text { at } y=h_{1} \\
\sigma-\gamma_{1} \sigma_{y}=1, \quad \text { at } y=h_{2}
\end{gathered}
$$

\section{Closed Form Solution of the Model}

In the present section, an effective procedure is introduced to obtain the analytical solutions for the resulted system of linear and nonlinear differential equations.

On integrating (6) twice and then inserting the resulted equation into (5), we obtain

$$
\frac{\partial^{2} \theta}{\partial y^{2}}+N_{b} f_{1}(x) \frac{\partial \theta}{\partial y}=0
$$

This equation can be exactly solved to give the temperature distribution, and therefore the nanoparticles concentration, as

$$
\begin{aligned}
\theta(x, y)= & f_{4}(x) e^{-N_{b} f_{1}(x) y}+\frac{1}{N_{b}} \frac{f_{3}(x)}{f_{1}(x)}, \\
\sigma(x, y)= & -\frac{N_{t}}{N_{b}} f_{4}(x) e^{-N_{b} f_{1}(x) y} \\
& +f_{1}(x) y+f_{2}(x)-\frac{N_{t}}{N_{b}^{2}} \frac{f_{3}(x)}{f_{1}(x)}
\end{aligned}
$$

where $f_{i}(x), i=1,2,3,4$ are unknown functions to be determined. On applying the boundary conditions (9) on (12), and then solving the resulted equations, we get

$$
\begin{aligned}
& f_{4}=\frac{1}{\left(1+\gamma N_{b} f_{1}\right) r_{2}^{f_{1}}-\left(1-\gamma N_{b} f_{1}\right) r_{1}^{f_{1}}}, \\
& f_{3}=\frac{-N_{b} f_{1}\left(1-\gamma N_{b} f_{1}\right) r_{1}^{f_{1}}}{\left(1+\gamma N_{b} f_{1}\right) r_{2}^{f_{1}}-\left(1-\gamma N_{b} f_{1}\right) r_{1}^{f_{1}}} .
\end{aligned}
$$

Further, applying the boundary conditions (10) on (13), and then solving the given system, results

$$
\begin{aligned}
f_{2}= & \frac{N_{t}}{N_{b}^{2}} \frac{f_{3}}{f_{1}}-\left(\gamma_{1} f_{1}-\frac{1}{N_{b}}\right) N_{t} f_{4} r_{1}^{f_{1}} \\
& -\left(\gamma_{1}+h_{1}\right) f_{1},
\end{aligned}
$$

where

$$
r_{1}=e^{-N_{b} h_{1}}, \quad r_{2}=e^{-N_{b} h_{2}}
$$

The above analysis leads to the following implicit algebraic equation in $f_{1}(x)$ :

$$
\begin{aligned}
\frac{N_{t}}{N_{b}}[ & \left.\frac{\left(\gamma_{1} N_{b} f_{1}-1\right) r_{1}^{f_{1}}+\left(\gamma_{1} N_{b} f_{1}+1\right) r_{2}^{f_{1}}}{\left(\gamma N_{b} f_{1}-1\right) r_{1}^{f_{1}}+\left(\gamma N_{b} f_{1}+1\right) r_{2}^{f_{1}}}\right] \\
& +\left(2 \gamma_{1}+h_{1}-h_{2}\right) f_{1}=-1 .
\end{aligned}
$$

\section{Exact Solutions of the Physical Variables}

4.1. Exact Expression of the Stream Function $\psi(x, y)$. Now, we search for the exact expression of the stream function $\psi(x, y)$. By integrating the $\psi(x, y)$ expression in (4) twice, we obtain

$$
\psi=f_{8}+f_{7} y+\frac{1}{2} f_{6} y^{2}+\frac{1}{6} f_{5} y^{3}+g(y)
$$

where

$$
\begin{gathered}
\Omega_{1}(x)=\left(\frac{\beta_{r} N_{t}}{N_{b}}-G_{r}\right) \frac{1}{N_{b}} \frac{f_{3}}{f_{1}}-\beta_{r} f_{2}, \\
\Omega_{2}(x)=\left(\frac{\beta_{r} N_{t}}{N_{b}}-G_{r}\right) f_{4}, \\
g(y)=\frac{1}{6} \Omega_{1} y^{3}-\frac{1}{24} \beta_{r} f_{1} y^{4}-\frac{\Omega_{2}}{\left(N_{b} f_{1}\right)^{3}} e^{-N_{b} f_{1} y} .
\end{gathered}
$$

Applying the boundary conditions (8) on the $\psi$-equation given in (18) and (19), we obtain the following system:

$$
\begin{gathered}
f_{8}+f_{7} h_{i}+\frac{1}{2} f_{6} h_{i}^{2}+\frac{1}{6} f_{5} h_{i}^{3}=R_{i}(x), \\
f_{7}+A\left(\beta+h_{1}\right) f_{6}+\left(\frac{1}{2} h_{i}^{2}+A \beta h_{i}\right) f_{5}=S_{i}(x),
\end{gathered}
$$

where

$$
\begin{gathered}
R_{i}(x)=\frac{A}{2} F-g\left(h_{i}\right), \\
S_{i}(x)=-1-g^{\prime}\left(h_{i}\right)-A \beta g^{\prime \prime}\left(h_{i}\right),
\end{gathered}
$$

where again $A=1,-1$ when $i=1,2$, respectively. 
4.1.1. Obtaining the Values of $f_{j}, j=5, \ldots, 8$. On solving the last linear system in (20) with (21), we obtain

$$
\begin{aligned}
& f_{5}=\frac{6\left(-2 R_{1}+2 R_{2}+\left(h_{1}-h_{2}\right)\left(S_{1}+S_{2}\right)\right)}{\left(h_{1}-h_{2}\right)^{2}\left(6 \beta+h_{1}-h_{2}\right)}, \\
& f_{6}=2\left(-h_{1}^{3}\left(S_{1}+2 S_{2}\right)\right. \\
& +3 h_{1}^{2}\left(R_{1}-R_{2}+\left(-2 \beta+h_{2}\right) S_{2}\right) \\
& +3 h_{1}\left(2 \beta R_{1}-2 \beta R_{2}\right. \\
& \left.+h_{2}\left(\left(-2 \beta+h_{2}\right) S_{1}+2 \beta S_{2}\right)\right) \\
& -h_{2}\left(3\left(-2 \beta+h_{2}\right) R_{1}+\left(6 \beta-3 h_{2}\right) R_{2}\right. \\
& \left.\left.+h_{2}\left(2\left(-3 \beta+h_{2}\right) S_{1}+h_{2} S_{2}\right)\right)\right) \\
& \times\left(\left(h_{1}-h_{2}\right)^{2}\left(2 \beta+h_{1}-h_{2}\right)\left(6 \beta+h_{1}-h_{2}\right)\right)^{-1}, \\
& f_{7}=\left(h_{1}^{4} S_{2}+h_{1}^{3}\left(2\left(-\beta+h_{2}\right) S_{1}+4 \beta S_{2}\right)\right. \\
& +2 h_{1}\left(3\left(2 \beta^{2}-4 \beta h_{2}+h_{2}^{2}\right)\left(R_{1}-R_{2}\right)\right. \\
& \left.+h_{2}^{2}\left(-3 \beta+h_{2}\right) S_{2}\right) \\
& +h_{2}\left(6 \beta\left(-2 \beta+h_{2}\right) R_{1}\right. \\
& +6 \beta\left(2 \beta-h_{2}\right) R_{2} \\
& \left.+h_{2}^{2}\left(\left(-4 \beta+h_{2}\right) S_{1}+2 \beta S_{2}\right)\right) \\
& +h_{1}^{2}\left(6\left(\beta-h_{2}\right) R_{1}\right. \\
& -3\left(2\left(\beta-h_{2}\right) R_{2}\right. \\
& \left.\left.\left.+h_{2}\left(\left(-2 \beta+h_{2}\right) S_{1}+h_{2} S_{2}\right)\right)\right)\right) \\
& \times\left(\left(h_{1}-h_{2}\right)^{2}\left(2 \beta+h_{1}-h_{2}\right)\left(6 \beta+h_{1}-h_{2}\right)\right)^{-1}, \\
& f_{8}=h_{2}^{2}\left(12 \beta^{2}-8 \beta h_{2}+h_{2}^{2}\right) R_{1} \\
& +h_{1}^{3}\left(2 \beta-h_{2}\right)\left(4 R_{2}+h_{2}\left(S_{1}-2 S_{2}\right)\right) \\
& +h_{1}^{4}\left(R_{2}-h_{2} S_{2}\right) \\
& +h_{1}^{2}\left(12 \beta^{2} R_{2}-6 \beta h_{2}\left(R_{1}+3 R_{2}\right)\right. \\
& +h_{2}^{3}\left(2 S_{1}-S_{2}\right) \\
& \left.+3 h_{2}^{2}\left(R_{1}+R_{2}-2 \beta S_{1}+2 \beta S_{2}\right)\right) \\
& -h_{1} h_{2}\left(2\left(6 \beta^{2}-9 \beta h_{2}+2 h_{2}^{2}\right) R_{1}\right. \\
& +6 \beta\left(2 \beta-h_{2}\right) R_{2} \\
& \left.+h_{2}^{2}\left(\left(-4 \beta+h_{2}\right) S_{1}+2 \beta S_{2}\right)\right) \\
& \times\left(\left(h_{1}-h_{2}\right)^{2}\left(2 \beta+h_{1}-h_{2}\right)\left(6 \beta+h_{1}-h_{2}\right)\right)^{-1} .
\end{aligned}
$$

4.2. Exact Expression of the Pressure Gradient $d p / d x$. To get the pressure gradient $d p / d x$, we obtain from (7) and the above analysis that

$$
\frac{d p}{d x}=\Omega_{3}(x)-\beta_{r} f_{1} y+\left(1+N_{b} f_{1}\right) \Omega_{2}(x) e^{-N_{b} f_{1} y}
$$

where

$$
\Omega_{3}(x)=\Omega_{1}(x)+f_{5}(x)+\beta_{r} f_{1}(x),
$$

and further all other functions are already well defined in the present section.

4.3. Numerical Values of $f_{1}(x)$. In Section 3, the general closed form solutions for the temperature distribution $\theta$ and nanoparticles concentration $\sigma$ are obtained and expressed in terms of $f_{1}, f_{2}, f_{3}$, and $f_{4}$. As $f_{2}, f_{3}$, and $f_{4}$ depend on the evaluating of $f_{1}$, it is noticed from (17) that $f_{1}$ is governed by a nonlinear algebraic equation. Once this equation is solved for $f_{1}$, the analytical expressions for $\theta$ and $\sigma$ are established.

It should be noted that obtaining the value of $f_{1}$ analytically from (17) in terms of the other parameters set is a very difficult task, and it may be impossible. However, with the help of MATHEMATICA 6 software, the numerical solutions are still available. Values for $f_{1}$ at some given cases are presented in Tables 1 and 2 . These obtained values for $f_{1}$ play an important role to get several plots for variation of the temperature distribution and nanoparticles concentration, which are introduced in the next section.

\section{Results and Discussion}

Besides discussing the effects of various physical parameters on the temperature distribution and nanoparticles concentration, comparing with the approximate solutions obtained by Akbar et al. [33] is also presented.

Effect of $N_{t}$ on the temperature profile $\theta$ for different values of the thermophoresis parameter $N_{t}$ is plotted in Figure 2 
TABLE 2: The numerical values of $f_{1}$ at different values of $\gamma$ and $\gamma_{1}$ for $a=0.1, d=1, b=0.5, x=1, \phi=0.2$ when $N_{b}=2$ for $N_{t}=0.8$ and 5 .

\begin{tabular}{|c|c|c|c|c|c|c|c|}
\hline$N_{t}$ & $\gamma_{1}$ & $\gamma$ & $f_{1}$ & $N_{t}$ & $\gamma$ & $\gamma_{1}$ & $f_{1}$ \\
\hline \multirow{4}{*}{0.8} & \multirow{4}{*}{0.5} & 0.2 & -0.468643 & \multirow{4}{*}{0.8} & \multirow{4}{*}{0.5} & 0.2 & -0.496497 \\
\hline & & 0.4 & -0.442588 & & & 0.4 & -0.451474 \\
\hline & & 0.6 & -0.424333 & & & 0.6 & -0.415994 \\
\hline & & 0.8 & -0.410694 & & & 0.8 & -0.387347 \\
\hline \multirow{4}{*}{5.0} & \multirow{4}{*}{0.5} & 0.2 & -1.520890 & \multirow{4}{*}{5.0} & \multirow{4}{*}{0.5} & 0.2 & -1.035780 \\
\hline & & 0.4 & -1.176000 & & & 0.4 & -1.067390 \\
\hline & & 0.6 & -1.010570 & & & 0.6 & -1.095500 \\
\hline & & 0.8 & -0.907813 & & & 0.8 & -1.120570 \\
\hline
\end{tabular}

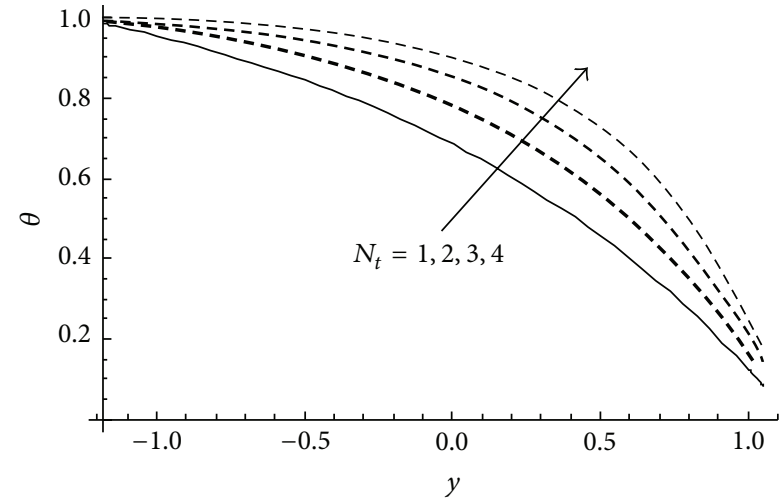

(a) $N_{b}=0.8$

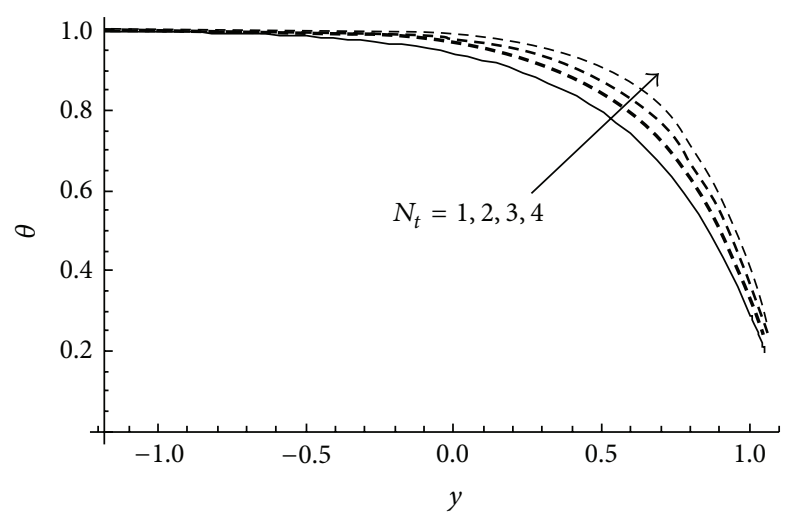

(b) $N_{b}=5$

Figure 2: Variation of the temperature profile $\theta$ at different values of $N_{t}$ for $a=0.1, d=1, b=0.5, x=1, \phi=0.2$ when $\gamma=\gamma_{1}=0.1$ at (a) $N_{b}=0.8$ and (b) $N_{b}=5$.

at two different values for Brownian motion parameter $N_{b}$. It is observed from this figure that the temperature profile increases when thermophoresis parameter $N_{t}$ increases for the small or high value of Brownian motion parameter $N_{b}$. It should be mentioned here that the present results are derived through exact solutions not as in [33] by an approximate way via the homotopy perturbation method. For the purpose of comparison, remarkable differences can be easily detected between our exact results presented in Figure 2(a) and those obtained by Akbar et al. [33] at the same values of the physical parameters. Regarding this we may point out that

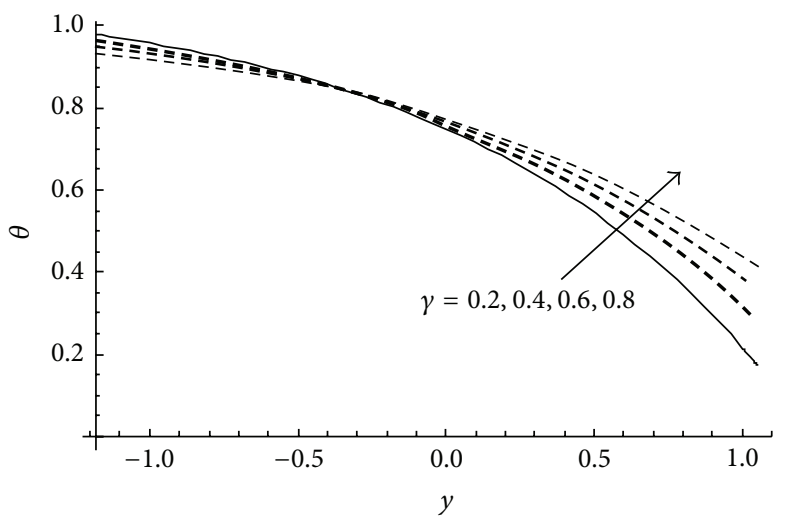

(a) $N_{t}=0.8$

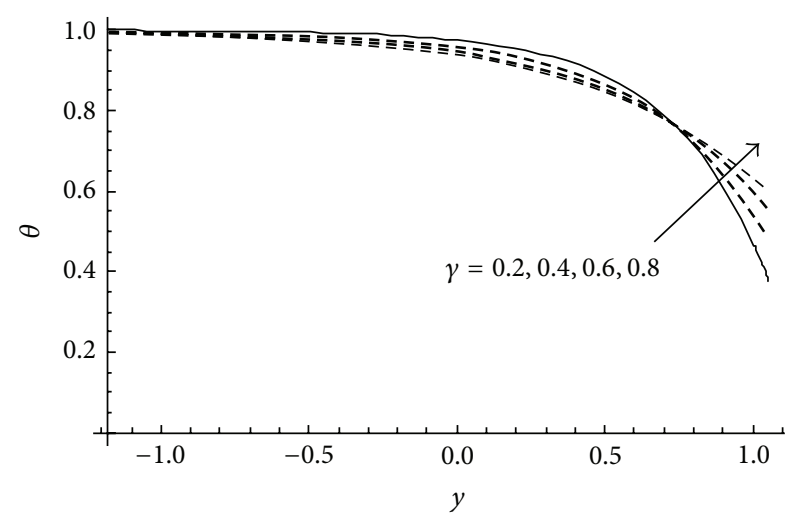

(b) $N_{t}=5$

FIGURE 3: Variation of the temperature profile $\theta$ at different values of $\gamma$ for $a=0.1, d=1, b=0.5, x=1, \phi=0.2, \gamma_{1}=0.5, N_{b}=2$ at (a) $N_{t}=0.8$ and (b) $N_{t}=5$.

the approximate solutions obtained in [33] were not effective enough to give the correct physical curves.

Figure 3 shows the effect of the slip parameter $\gamma$ on the temperature profile $\theta$ at two different values of the thermophoresis parameter $N_{t}$. The results reveal that the temperature profile decreases in a specific domain with increasing $\gamma$ for any small or high value of $N_{t}$. After that domain, the behaviour of $\theta$ is different, where it increases with increasing $\gamma$. However, the domain in which the temperature profile decreases with increasing $\gamma$ becomes wider when $N_{t}$ takes high values; see Figure 3(b). 


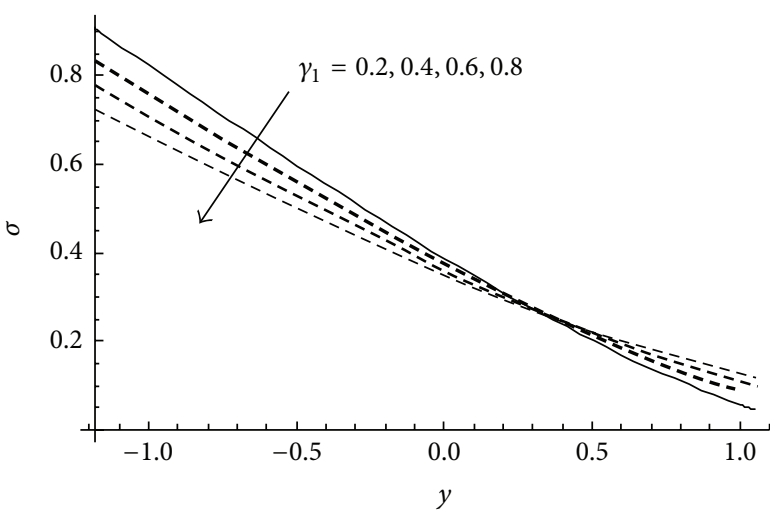

(a) $N_{b}=0.8$

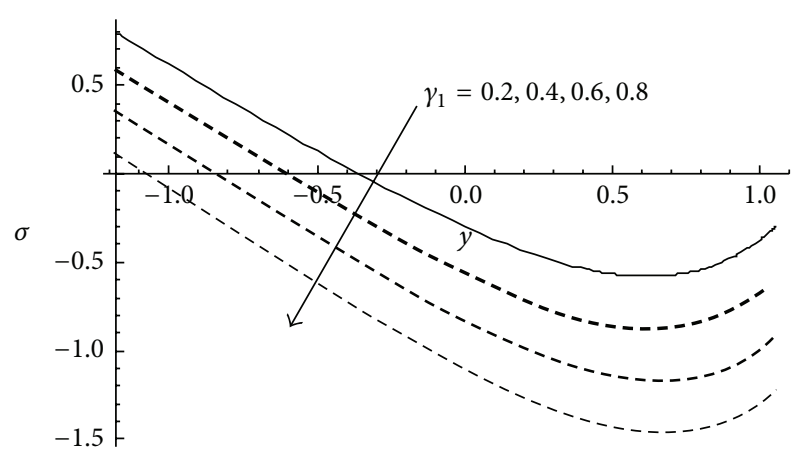

(b) $N_{b}=5$

FIGURE 4: Variation profile of nanoparticles concentration $\sigma$ at different values of $\gamma_{1}$ for $a=0.1, d=1, b=0.5, x=1, \phi=0.2, \gamma=0.5$, $N_{b}=2$ at (a) $N_{t}=0.8$ and (b) $N_{t}=5$.

The nanoparticles concentration $\sigma$ is depicted in Figures 4 and 5. In Figure 4(a) when the small value of Brownian motion parameter $N_{b}$ is presented, it is observed that the nanoparticles concentration $\sigma$ decreases in a certain domain with increasing the slip parameter $\gamma_{1}$. A converse of this behaviour occurs after that domain. At a higher value of Brownian motion parameter $N_{b}$, it is noticed that the nanoparticles concentration $\sigma$ decreases in the whole domain with increasing the slip parameter $\gamma_{1}$. On comparing the results depicted in Figure 4(a) and those obtained by Akbar et al. [33] at the same values of the physical parameters, slight differences are observed. Therefore, the current exact solutions, which can be verified by direct substitution into the governing differential equations and the boundary conditions, are reported in this paper for the first time. In addition, the effect of the thermophoresis parameter $N_{t}$ on the nanoparticles concentration $\sigma$ is depicted in Figure 5 at two different values for $N_{b}$. The behaviour of $\sigma$ always decreases with increasing $N_{t}$. However, slight differences are also observed between the current results in Figure 5(b) and those obtained in Figure 4(b) by Akbar et al. [33]. Accordingly, the present results can be viewed as optimal and more accurate.

\section{Conclusion}

In this paper, exact effects of the slip conditions and peristaltic action on the nanofluid flow in an asymmetric channel were

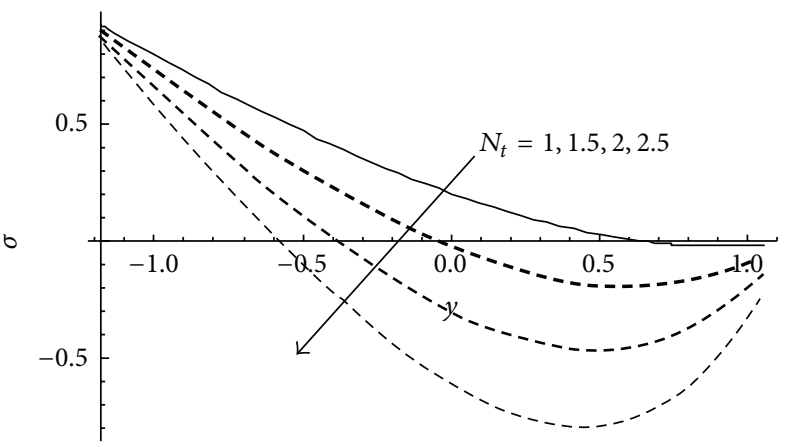

(a) $N_{b}=0.8$

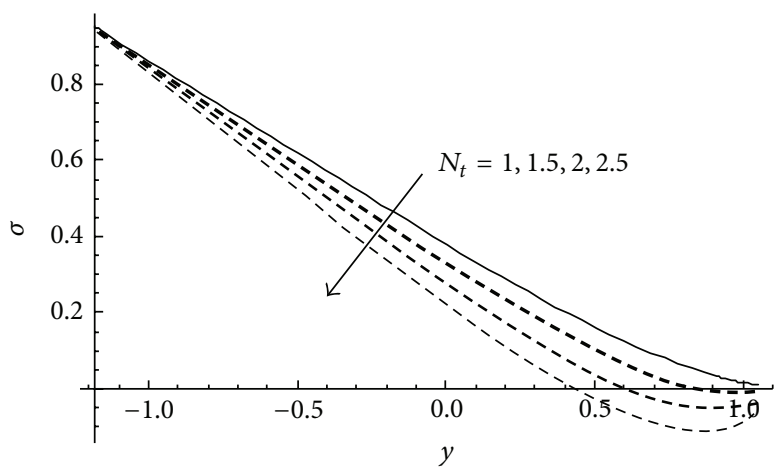

(b) $N_{b}=5$

FIGURE 5: Variation profile of nanoparticles concentration $\sigma$ at different values of $N_{b}$ for $a=0.1, d=1, b=0.5, x=1, \phi=0.2$ when $\gamma=\gamma_{1}=0.1$ at (a) $N_{b}=0.8$ and (b) $N_{b}=5$.

discussed for the variations of the temperature profile and nanoparticles concentration. The flow was described by a system of linear and nonlinear partial differential equations with complex boundary conditions generated on the flexible walls of the channel.

The exact solutions have been successfully obtained and reported for the first time. In addition, the obtained exact numerical results for effects of the slip condition, thermophoresis, and Brownian motion parameters on the temperature and nanoparticles concentration profiles show slight differences on comparing with the approximate solutions obtained via the homotopy perturbation method. The current analysis may throw some light on the nanofluid dynamic aspects used in the biomedical applications to treat the cancer's tissues, with the help of magnetic nanoparticles under the peristalsis on the blood vessels.

\section{Conflict of Interests}

The authors declare that there is no conflict of interests regarding the publication of this paper.

\section{References}

[1] T. W. Latham, Fluid motion in a peristaltic pump [M.S. thesis], MIT, Cambridge, UK, 1966. 
[2] T.-F. Zien and S. Ostrach, "A long wave approximation to peristaltic motion," Journal of Biomechanics, vol. 3, no. 1, pp. 63$75,1970$.

[3] J.-S. Lee and Y.-C. Fung, "Flow in nonuniform small blood vessels," Microvascular Research, vol. 3, no. 3, pp. 272-287, 1971.

[4] L. M. Srivastava, V. P. Srivastava, and S. N. Sinha, "Peristaltic transport of a physiological fluid. Part I. Flow in non-uniform geometry," Biorheology, vol. 20, no. 2, pp. 153-166, 1983.

[5] S. Takabatake, K. Ayukawa, and A. Mori, "Peristaltic pumping in circular cylindrical tubes: a numerical study of fluid transport and its efficiency," Journal of Fluid Mechanics, vol. 193, pp. 267283, 1988.

[6] E. F. El Shehawey and K. S. Mekheimer, "Couple-stresses in peristaltic transport of fluids," Journal of Physics D, vol. 27, no. 6, pp. 1163-1170, 1994.

[7] R. A. Ramachandra and S. Usha, "Peristaltic transport of two immiscible viscous fluids in a circular tube," Journal of Fluid Mechanics, vol. 298, pp. 271-285, 1995.

[8] K. S. Mekheimer, "Peristaltic transport of a couple stress fluid in a uniform and non-uniform channels," Biorheology, vol. 39, no. 6, pp. 755-765, 2002.

[9] K. Vajravelu, S. Sreenadh, and V. R. Babu, "Peristaltic transport of a Herschel-Bulkley fluid in an inclined tube," International Journal of Non-Linear Mechanics, vol. 40, no. 1, pp. 83-90, 2005.

[10] K. S. Mekheimer and Y. Abd elmaboud, "The influence of heat transfer and magnetic field on peristaltic transport of a Newtonian fluid in a vertical annulus: application of an endoscope," Physics Letters A, vol. 372, no. 10, pp. 1657-1665, 2008.

[11] K. S. Mekheimer and Y. Abd elmaboud, "Peristaltic flow of a couple stress fluid in an annulus: application of an endoscope," Physica A, vol. 387, no. 11, pp. 2403-2415, 2008.

[12] K. De Vries, E. A. Lyons, G. Ballard, C. S. Levi, and D. J. Lindsay, "Contractions of the inner third of the myometrium," American Journal of Obstetrics and Gynecology, vol. 162, no. 3, pp. 679-682, 1990.

[13] O. Eytan, A. J. Jaffa, and D. Elad, "Peristaltic flow in a tapered channel: application to embryo transport within the uterine cavity," Medical Engineering and Physics, vol. 23, no. 7, pp. 473482, 2001.

[14] E. F. Elshehawey, N. T. Eldabe, E. M. Elghazy, and A. Ebaid, "Peristaltic transport in an asymmetric channel through a porous medium," Applied Mathematics and Computation, vol. 182, no. 1, pp. 140-150, 2006.

[15] M. V. Subba Reddy, A. Ramachandra Rao, and S. Sreenadh, "Peristaltic motion of a power-law fluid in an asymmetric channel," International Journal of Non-Linear Mechanics, vol. 42, no. 10, pp. 1153-1161, 2007.

[16] A. Ebaid, "Effects of magnetic field and wall slip conditions on the peristaltic transport of a Newtonian fluid in an asymmetric channel," Physics Letters A, vol. 372, no. 24, pp. 4493-4499, 2008.

[17] A. M. Sobh, "Slip flow in peristaltic transport of a Carreau fluid in an asymmetric channel," Canadian Journal of Physics, vol. 87, no. 8, pp. 957-965, 2009.

[18] G. C. Shit, M. Roy, and E. Y. K. Ng, "Effect of induced magnetic field on peristaltic flow of a micropolar fluid in an asymmetric channel," International Journal for Numerical Methods in Biomedical Engineering, vol. 26, no. 11, pp. 13801403, 2010.

[19] K. S. Mekheimer, S. Z. A. Husseny, and Y. Abd Elmaboud, "Effects of heat transfer and space porosity on peristaltic flow in a vertical asymmetric channel," Numerical Methods for Partial Differential Equations, vol. 26, no. 4, pp. 747-770, 2010.

[20] S. Srinivas and R. Muthuraj, "Effects of chemical reaction and space porosity on MHD mixed convective flow in a vertical asymmetric channel with peristalsis," Mathematical and Computer Modelling, vol. 54, no. 5-6, pp. 1213-1227, 2011.

[21] S. Srinivas, R. Gayathri, and M. Kothandapani, "Mixed convective heat and mass transfer in an asymmetric channel with peristalsis," Communications in Nonlinear Science and Numerical Simulation, vol. 16, no. 4, pp. 1845-1862, 2011.

[22] K. Das, "Influence of slip and heat transfer on mhd peristaltic flow of a jeffrey fluid in an inclined asymmetric porous channel," Indian Journal of Mathematics, vol. 54, pp. 19-45, 2012.

[23] Y. Abd Elmaboud, S. Kh. Mekheimer, and A. I. Abdellateef, "Thermal properties of couple-stress fluid flow in an asymmetric channel with peristalsis," Journal of Heat Transfer, vol. 135, no. 4, 8 pages, 2013.

[24] N. S. Akbar and S. Nadeem, "Thermal and velocity slip effects on the peristaltic flow of a six constant Jeffrey's fluid model," International Journal of Heat and Mass Transfer, vol. 55, no. 1516, pp. 3964-3970, 2012.

[25] N. S. Akbar and S. Nadeem, "Numerical and analytical simulation of the peristaltic flow of Jeffrey fluid with Reynold's model of viscosity," International Journal of Numerical Methods for Heat \& Fluid Flow, vol. 22, pp. 458-472, 2012.

[26] N. S. Akbar, S. Nadeem, T. Hayat, and A. A. Hendi, "Analytical and numerical analysis of Vogel's model of viscosity on the peristaltic flow of Jeffrey fluid," Journal of Aerospace Engineering, vol. 25, no. 1, pp. 64-70, 2012.

[27] N. S. Akbar and S. Nadeem, "Analytical and numerical study of peristaltic transport of a Johnson-Segalman fluid in an endoscope," Chinese Physics B, vol. 22, no. 1, Article ID 014703, 2013.

[28] N. S. Akbar and S. Nadeem, "Mixed convective magnetohydrodynamic peristaltic flow of a Jeffrey nanofluid with Newtonian heating," Zeitschrift für Naturforschung, vol. 68, pp. 433-441, 2013.

[29] N. S. Akbar and S. Nadeem, "Endoscopic effects on peristaltic flow of a nanofluid," Communications in Theoretical Physics, vol. 56, no. 4, pp. 761-768, 2011.

[30] N. S. Akbar, S. Nadeem, T. Hayat, and A. A. Hendi, "Peristaltic flow of a nanofluid in a non-uniform tube," Heat and Mass Transfer/Waerme-und Stoffuebertragung, vol. 48, no. 2, pp. 451459, 2012.

[31] N. S. Akbar and S. Nadeem, "Peristaltic flow of a Phan-ThienTanner nanofluid in a diverging tube," Heat Transfer, vol. 41, no. 1, pp. 10-22, 2012.

[32] M. Mustafa, S. Hina, T. Hayat, and A. Alsaedi, "Influence of wall properties on the peristaltic flow of a nanofluid: analytic and numerical solutions," International Journal of Heat and Mass Transfer, vol. 55, pp. 4871-4877, 2012.

[33] N. S. Akbar, S. Nadeem, T. Hayat, and A. A. Hendi, "Peristaltic flow of a nanofluid with slip effects," Meccanica, vol. 47, pp. 12831294, 2012.

[34] O. A. Bég and D. Tripathi, "Mathematica simulation of peristaltic pumping with double-diffusive convection in nanofluids: a bio-nano-engineering model," Journal of Nanoengineering and Nanosystems, 2012.

[35] S. U. S. Choi, "Enhancing thermal conductivity of fluids with nanoparticles," in The Proceedings of the ASME International Mechanical Engineering Congress and Exposition, ASME, San Francisco, Calif, USA. 
[36] S. U. S. Choi, Z. G. Zhang, W. Yu, F. E. Lockwood, and E. A. Grulke, "Anomalous thermal conductivity enhancement in nanotube suspensions," Applied Physics Letters, vol. 79, no. 14, pp. 2252-2254, 2001.

[37] Q. A. Pankhurst, J. Connolly, S. K. Jones, and J. Dobson, "Applications of magnetic nanoparticles in biomedicine," Journal of Physics D, vol. 36, no. 13, pp. R167-R181, 2003.

[38] M. R. Habibi, M. Ghassemi, and M. H. Hamedi, "Analysis of high gradient magnetic field effects on distribution of nanoparticles injected into pulsatile blood stream," Journal of Magnetism and Magnetic Materials, vol. 324, no. 8, pp. 14731482, 2012.

[39] M. Majumder, N. Chopra, R. Andrews, and B. J. Hinds, "Nanoscale hydrodynamics: enhanced flow in carbon nanotubes," Nature, vol. 438, no. 7064, p. 44, 2005.

[40] M. Mishra and A. R. Rao, "Peristaltic transport of a Newtonian fluid in an asymmetric channel," Zeitschrift fur Angewandte Mathematik und Physik, vol. 54, no. 3, pp. 532-550, 2003. 


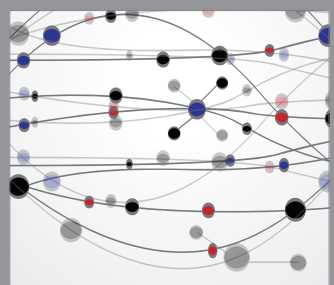

The Scientific World Journal
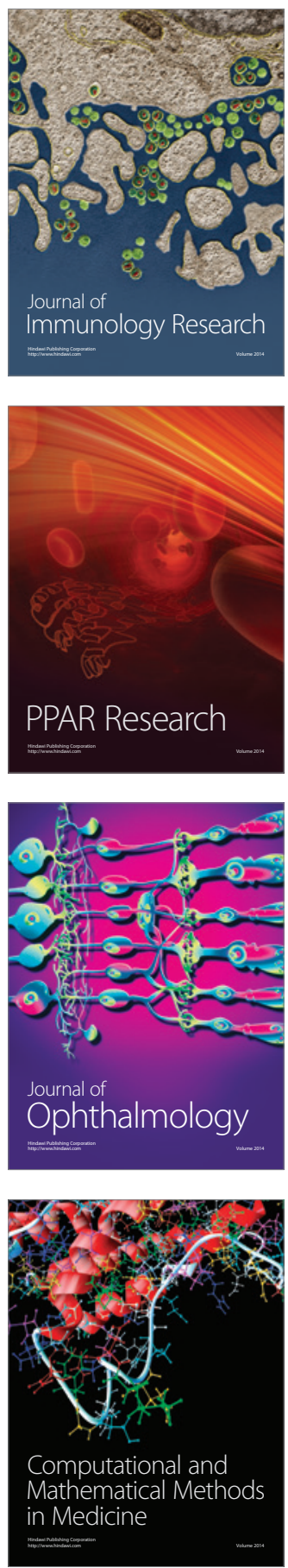

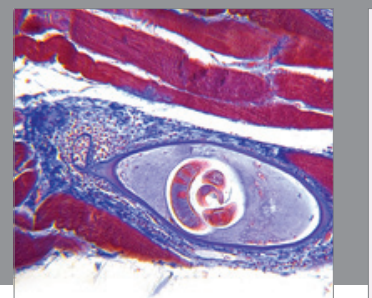

Gastroenterology

Research and Practice
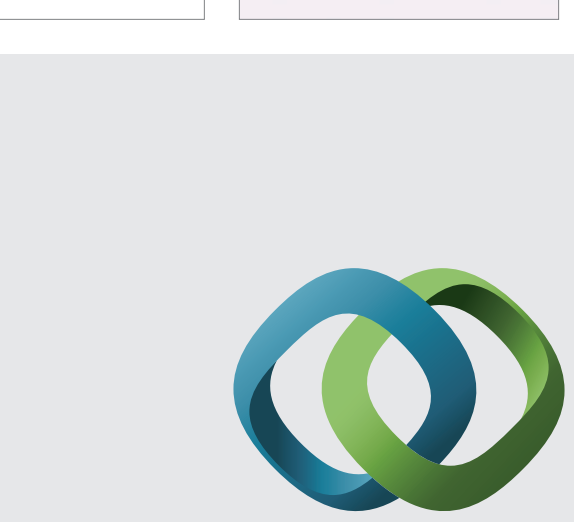

\section{Hindawi}

Submit your manuscripts at

http://www.hindawi.com
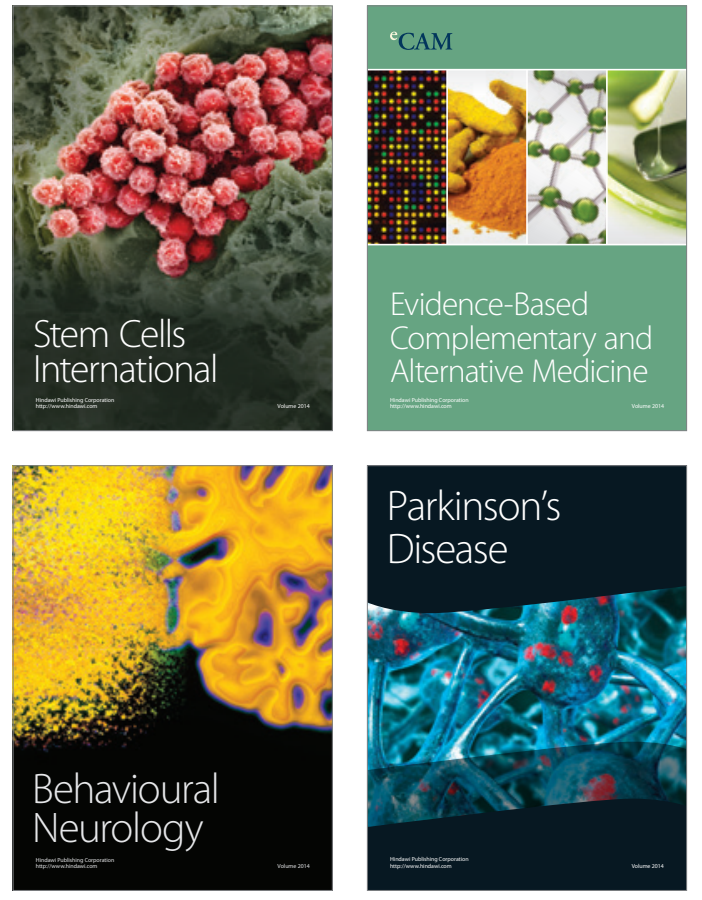
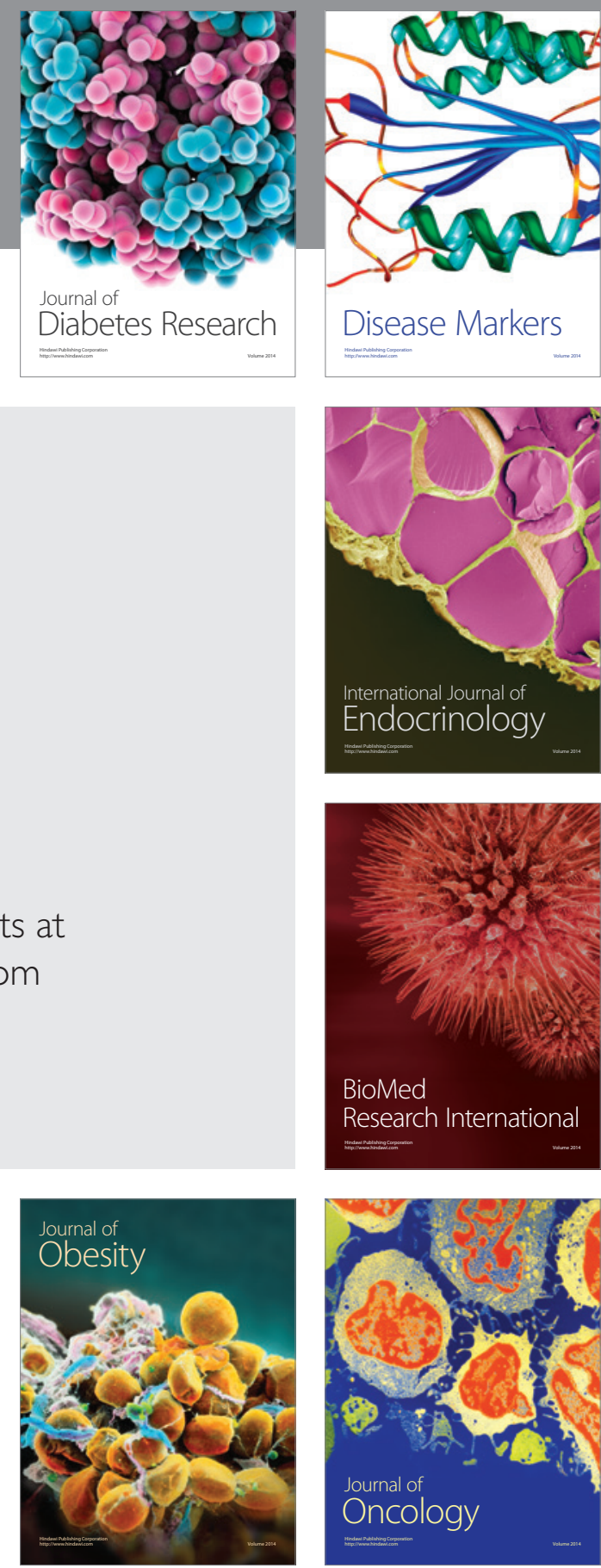

Disease Markers
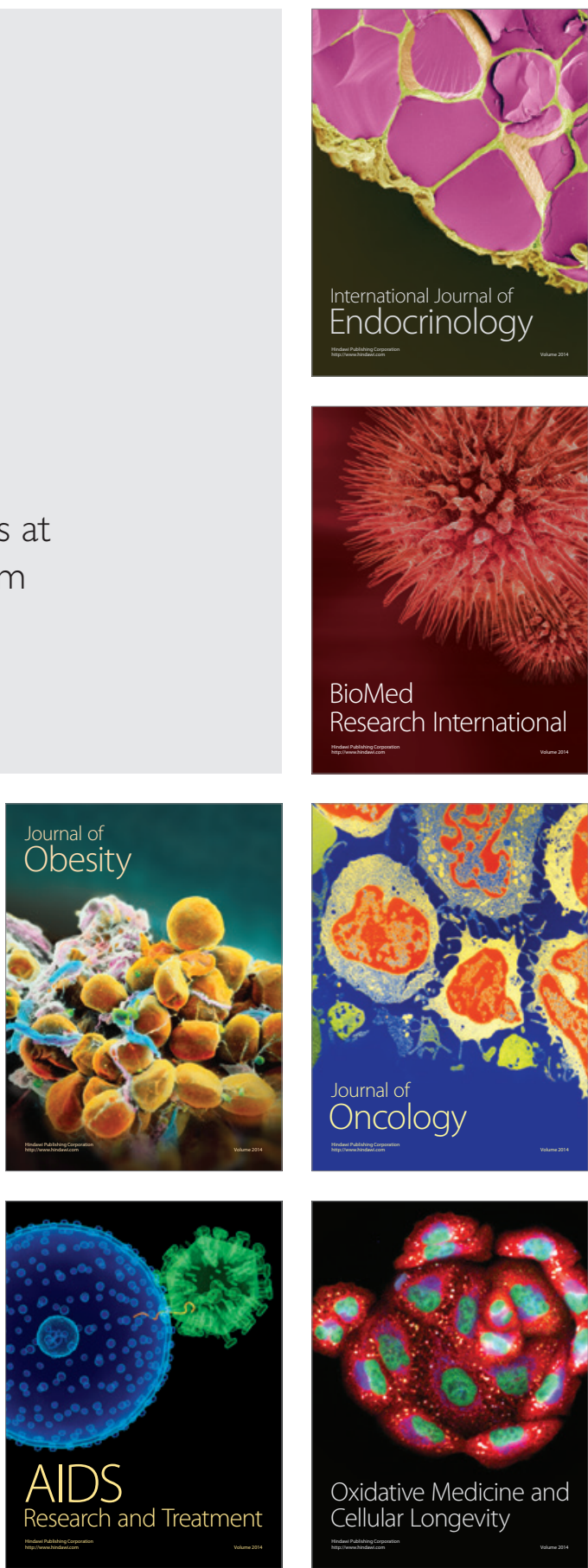